\title{
Fungi Treated with Small Chemicals Exhibit Increased Antimicrobial Activity against Facultative Bacterial and Yeast Pathogens
}

\author{
Christoph Zutz, ${ }^{1}$ Dragana Bandian, ${ }^{2}$ Bernhard Neumayer, ${ }^{1,3}$ Franz Speringer, ${ }^{3}$ \\ Markus Gorfer, ${ }^{2,3}$ Martin Wagner, ${ }^{1}$ Joseph Strauss, ${ }^{2,3}$ and Kathrin Rychli ${ }^{1}$ \\ ${ }^{1}$ Institute for Milk Hygiene, University of Veterinary Medicine Vienna, Veterinaerplatz 1, 1210 Vienna, Austria \\ ${ }^{2}$ AIT-Austrian Institute of Technology GmbH, University and Research Campus Tulln, Konrad Lorenz Straße 24, \\ 3430 Tulln on the Danube, Austria \\ ${ }^{3}$ Fungal Genetics and Genomics Unit, Department of Applied Genetics and Cell Biology, BOKU University of Natural Resources and \\ Life Sciences Vienna, Konrad Lorenz Straße 24, 3430 Tulln on the Danube, Austria
}

Correspondence should be addressed to Kathrin Rychli; kathrin.rychli@vetmeduni.ac.at

Received 7 March 2014; Revised 16 June 2014; Accepted 18 June 2014; Published 9 July 2014

Academic Editor: Isabel Sá-Correia

Copyright (C) 2014 Christoph Zutz et al. This is an open access article distributed under the Creative Commons Attribution License, which permits unrestricted use, distribution, and reproduction in any medium, provided the original work is properly cited.

\begin{abstract}
For decades, fungi have been the main source for the discovery of novel antimicrobial drugs. Recent sequencing efforts revealed a still high number of so far unknown "cryptic" secondary metabolites. The production of these metabolites is presumably epigenetically silenced under standard laboratory conditions. In this study, we investigated the effect of six small mass chemicals, of which some are known to act as epigenetic modulators, on the production of antimicrobial compounds in 54 spore forming fungi. The antimicrobial effect of fungal samples was tested against clinically facultative pathogens and multiresistant clinical isolates. In total, 30 samples of treated fungi belonging to six different genera reduced significantly growth of different test organisms compared to the untreated fungal sample (growth log reduction 0.3-4.3). For instance, the pellet of Penicillium restrictum grown in the presence of butyrate revealed significant higher antimicrobial activity against Staphylococcus (S.) aureus and multiresistant S. aureus strains and displayed no cytotoxicity against human cells, thus making it an ideal candidate for antimicrobial compound discovery. Our study shows that every presumable fungus, even well described fungi, has the potential to produce novel antimicrobial compounds and that our approach is capable of rapidly filling the pipeline for yet undiscovered antimicrobial substances.
\end{abstract}

\section{Introduction}

For decades, one of the main sources for the discovery of novel antimicrobial drugs has been the screening of fungal cultures for bioactive natural compounds $[1,2]$. However, due to the increasing risk of rediscovery of antimicrobial compounds, many screening programs have been stopped in the last decade. Nevertheless, the increasing rate of appearance of resistant pathogens, the decreasing lifetime of antibiotics in clinical use, and the decline of novel antibiotic candidates in the pipeline raise the necessity to develop new strategies for antimicrobial compound discovery [3]. In fungi, antimicrobial compounds are mainly produced during the secondary metabolism (SM). Ongoing genome sequencing of fungi leads to new insights into the quantity of genes related to the SM $[4,5]$. There is evidence that the number of genes involved in the SM is higher than the number of characterized fungal secondary metabolites, thus indicating a high number of so far unknown compounds $[4,6]$. Genes involved in the SM of fungi are often organized in gene clusters, which are mainly in a transcriptional silenced state, if fungi are grown under standard laboratory culture conditions [6]. These silenced gene clusters hold the potential to code for proteins involved in the biosynthesis of new antimicrobial compounds [7]. Several approaches to activate such "cryptic" gene clusters have been performed, for example, by transover-expressing cluster-resident transcription factor [8], by deleting precursor-consuming pathways [9], by cocultivation 
with bacteria [10], and by interference with the epigenetic silencing pathway [11]. The silencing of gene clusters has been linked to epigenetic mechanisms which influence the formation of "facultative heterochromatin" [12]. Two of the major epigenetic signals of chromatin regulation are acetylation of histones, which results mainly in activation of gene transcription, and methylation of histones and DNA, which is mainly linked to silencing of transcription [13]. Recent studies showed that various low molecular mass chemicals, in this study named small chemicals (SCs), are able to inhibit histone deacetylases (HDACs) and DNA methyltransferases (DNMTs) in fungi [14]. Furthermore, it was demonstrated in fungi that specific SCs influence the secondary metabolite profile $[15,16]$.

The aim of this study was to investigate the effect of different SCs on the production of antimicrobial compounds in 54 spore forming fungi. We used six SCs, of which three were HDAC inhibitors: valproic acid (VPA), which inhibits class I HDACs and induces proteosomal degradation of class II HDACs $[17,18]$; the antifungal compound trichostatin A (TSA), which inhibits class I and II HDAC activity [19]; and sodium butyrate (butyrate), which induces differentiation in eukaryotic cell lines via HDAC inhibition [2022]. In addition, we used the DNA methylation inhibitor 5-azacytidine (AZA), which incorporates into RNA and to less extend into DNA, thus leading to elevated levels of hypomethylated DNA $[23,24]$. Furthermore, we included Nacetyl-D-glucosamine (GlcNAc), which induces secondary metabolite production on Streptomyces spp. [25] and nitric oxide (NO) a phytohormone, which has been shown to regulate gene expression in fungi and might therefore play a role in SM regulation $[26,27]$.

\section{Materials and Methods}

2.1. Bacterial and Fungal Strains Used in This Study. Antimicrobial activity of fungal cultures was tested against the Gram positive and Gram negative facultative pathogens Staphylococcus (S.) aureus (Rosenbach 1884, ATCC6538) and Pseudomonas (P.) aeruginosa (Migula 1900, ATCC9027) and the yeast pathogens Candida (C.) albicans (Robin Berkhout, ATCC10231); all are obtained from DSMZ, Germany. For detailed analysis in an enhanced screen, we used Klebsiella pneumonia ATCC13883, Escherichia coli MC1061, the yeast-like potential pathogenic fungus Exophiala (E.) dermatitidis (internal strain collection number 132), and three multiresistant clinical isolates: extended-spectrum betalactamase (ESBL) Klebsiella (K.) pneumonia (B100173), ESBL Escherichia (E.) coli (B300129), and methicillin-resistant S. aureus (MRSA) B337919 (kindly provided by Analyse Biolab $\mathrm{GmbH}$, Austria). Initial cultivation was performed on tryptone soya agar (Oxoid, USA) for $18 \mathrm{~h}$ at $37^{\circ} \mathrm{C}$. One single colony was inoculated in tryptone soya broth supplemented with $6 \mathrm{~g} / \mathrm{L}$ yeast extract (TSBY, Oxoid, USA) and incubated at $37^{\circ} \mathrm{C}$ for $6 \mathrm{~h}$ for S. aureus and P. aeruginosa and for $10 \mathrm{~h}$ for $C$. albicans. All fungal strains used in this study were obtained from the fungal strain collection of the AIT (Austrian Institute of Technology, Fungal Genetics and Genomics Unit,
Table 1). Identification of the fungi was performed according to Klaubauf et al. [28].

2.2. Small Chemicals. SC (all Sigma Aldrich, USA) stock solutions were prepared as recently described [16]. Shortly, valproic acid (VPA, 2-propylpentanoic acid) was dissolved in $96 \%$ ethanol to a final concentration of $60 \mu \mathrm{M}$. Trichostatin A (TSA, 7-4-(dimethylamino)phenyl-N-hydroxy-4,6dimethyl-7-oxohepta-2,4-dienamide, $0.5 \mu \mathrm{M})$, sodium butyrate (butyrate, $9 \mu \mathrm{M}$ ), $5^{\prime}$-azacytidine (AZA, 4-amino$1-\beta$-D-ribofuranosyl-1,3,5-triazin-2(1H)-1, $2 \mu \mathrm{M}), \quad$ and $\mathrm{N}$-acetyl-D-glucosamine (GlcNAc, $0.5 \mu \mathrm{M}$ ) were dissolved in PBS ( $\mathrm{pH} 7$ ). To prepare a stock solution that releases nitric oxide, a stock solution of $\mathrm{NaNO}_{2}(0.3 \mathrm{M}$ in PBS) was mixed at a ratio 1 to 1 with acidified Moser medium ( $\mathrm{pH}$ 5.0). Stock solutions were stored at $-20^{\circ} \mathrm{C}$ for not more than 6 months.

2.3. Preparation of Fungal Cultures. The fungal strains used in this study were thawed from cryogenic storage vials, streaked on malt extract agar plates (MEA; Oxoid, USA), and grown at room temperature (RT) until sporulation took place. To harvest the spores, the agar plate was drenched with PBS supplemented with $0.1 \%$ tween and scraped with a sterile cotton stick. Purity of spore solution was assessed (Nikon, Eclipse E200 40x magnification) and spores were incubated a second time on MEA plates. After incubation at RT for 10 (8 and 20) days, the spores were harvested and inoculated at a concentration of $10^{6}$ spores $/ \mathrm{mL}$ in $20 \mathrm{~mL}$ minimal fungal medium (Moser medium, composed of $10 \mathrm{~g} / \mathrm{L}$ glucose, $0.2 \mathrm{~g} / \mathrm{L}$ yeast extract, $2 \mathrm{~g} / \mathrm{L}$ tryptic-digested peptone from casein, $0.5 \mathrm{~g} / \mathrm{L} \mathrm{KH}_{2} \mathrm{PO}_{4}, 50 \mathrm{mg} / \mathrm{L}$ inositol, $75 \mathrm{mg} / \mathrm{L} \mathrm{CaCl}, 10 \mathrm{mg} / \mathrm{L}$ $\mathrm{FeCl}_{3}, 150 \mathrm{mg} / \mathrm{L} \mathrm{MgSO}_{4}$, and $10 \mathrm{mg} / \mathrm{L} \mathrm{MnSO}_{4}$ ).

The fungal strains were grown in absence and presence of the different SCs (final concentration of $5 \mu \mathrm{M}$, except $1.5 \mathrm{mM}$ for NO) at RT for $72 \mathrm{~h}$ in the absence of light on a rotary shaker at $180 \mathrm{rpm}$.

Of each fungal culture, four different fractions were prepared: supernatant, extracted supernatant, pellet, and extracted pellet. The supernatant and mycelium were harvested using a sterile cellulose filter. The supernatant was furthermore filtered a second time through a cellulose filter and an aliquot was directly used in the antimicrobial activity screen (supernatant).

To prepare the extracted supernatant, the remaining supernatant was extracted with acetone $1: 1(\mathrm{v} / \mathrm{v})$ and agitated for $1 \mathrm{~h}$ at room temperature followed by extraction with chloroform 1:1 (v/v) and agitated for $30 \mathrm{~min}$. The extract was incubated at $4^{\circ} \mathrm{C}$ until complete separation of the organic and the water phase took place. The chloroform phase was transferred into a new tube, dried under reduced pressure in a centrifugal vacuum concentrator (Mivac, Fisher Scientific, USA), and resuspended in $1 \mathrm{~mL}$ Moser medium supplemented with 10\% DMSO (Sigma Aldrich, USA).

The fungal mycelium was dried between two sheets of Whatman filter paper (VWR, Germany). For the preparation of the pellet extract, $200 \mathrm{mg}$ of the mycelium was mixed with $1 \mathrm{~mL}$ PBS and $1 \mathrm{~g}$ of glass beads of 2 different diameters $(\emptyset 0.75$ and $1 \mathrm{~mm}$, Fisher Scientific, USA). Fungal cells were lysed 
TABLE 1: Fungal strains used in this study.

\begin{tabular}{|c|c|c|c|c|}
\hline Species & Internal strain code & Isolation source & Country & Sequencing $^{\circ}$ \\
\hline Aspergillus clavatus & L19 & Air sample & Austria & ITS-LSU \\
\hline Aspergillus flavipes & NG_p18 & Grassland soil & Austria; Riederberg & ITS \\
\hline Aspergillus flavus & L16 & Air sample & Austria & ITS-LSU \\
\hline Aspergillus niger & D_An & Indoor air & Austria & ITS-LSU \\
\hline Aspergillus nomius & E06 & & & ITS-LSU \\
\hline Aspergillus reptans & D_D37 & Indoor air & Austria & ITS-LSU \\
\hline Aspergillus sclerotiorum (section circumdati) & L07 & Air sample & Austria & ITS-LSU \\
\hline Aspergillus spp. & RJW & & Austria; Vienna & ITS \\
\hline Aspergillus sydowii & EVU-01 & Indoor wall & Austria; Vienna & ITS \\
\hline Aspergillus terreus & ÖFI 05-01B & & & ITS \\
\hline Aspergillus versicolor & MB0839-7 & Indoor air car & Austria & ITS-LSU \\
\hline Aspergillus westerdijkiae & D_At & Indoor air & Austria & ITS-LSU \\
\hline Cladosporium sphaerospermum & D_D48 & Indoor air & Austria & ITS-LSU \\
\hline Clavicipitaceae spp. & NG_p36 & Agricultural soil & & ITS \\
\hline Hypocrea koningii & NG_14 BRE-1P1 & Agricultural soil & Austria; Maissau & ITS \\
\hline Hypocrea lixii & NG_p16 & Agricultural soil & Austria; Niederschleinz & ITS \\
\hline Isaria farinosa & KF0909_H8 & Indoor material sample & Austria & ITS-LSU \\
\hline Lecythophora hoffmannii & NG_p46 & Agricultural soil & Austria; Maissau & ITS \\
\hline Oidiodendron cerealis & NG_p39 & Agricultural soil & Austria; Maissau & ITS \\
\hline Penicillium alberechii & Hbs-K14 & Air sample & Austria; Vienna & ITS \\
\hline Penicillium biourgeianum & GAbP & Indoor air & Germany & ITS \\
\hline Penicillium brevicompactum & Hbs-K13 & Air sample & Austria; Vienna & ITS \\
\hline Penicillium canescens & NG_p02 & Grassland soil & Austria; Riederberg & ITS \\
\hline Penicillium corylophilum & D_D42 & Indoor air & Austria & ITS-LSU \\
\hline Penicillium chrysogenum & MC-A12 & Silica plates air condition & Austria; Vienna & ITS \\
\hline Penicillium commune & MCB11 & Silica plates air condition & Austria; Vienna & ITS \\
\hline Penicillium crustosum & D_D27 & Indoor air & Austria & ITS-LSU \\
\hline Penicillium decumbens & D_D54 & Food sample & Austria & ITS-LSU \\
\hline Penicillium decaturense & RSF-Q205 & Root sample Quercus & Austria; Redlschlag & ITS \\
\hline Penicillium echinulatum & W121 & Warburgia ugandensis & Uganda & ITS-LSU \\
\hline Penicillium funiculosum & NG_p14 & Agricultural soil & & ITS \\
\hline Penicillium glabra & D_D28 & Indoor air & Austria & ITS-LSU \\
\hline Penicillium glabrum/thomii & Li0102II & Spruce needle & Austria; Kalwang & ITS-LSU \\
\hline Penicillium glandicola & NG_24 & Agricultural soil & Austria; Maissau & ITS \\
\hline Penicillium islandicum & NG_P43 & Agricultural soil & Austria; Riederberg & ITS \\
\hline Penicillium islandicum/rugulosum & NG_P23 & Agricultural soil & & ITS \\
\hline Penicillium janthinellum & NG_23 & Agricultural soil & Austria; Purkersdorf & ITS \\
\hline Penicillium lividum & Li0102XII & Spruce needle & Austria; Kalwang & ITS-LSU \\
\hline Penicillium miczynskii & $\mathrm{A} 04$ & Air sample & Austria & ITS-LSU \\
\hline Penicillium olsonii & D_D38 & Indoor air & Austria & ITS-LSU \\
\hline Penicillium piceum & D_D04 & Indoor air & Austria & ITS-LSU \\
\hline Penicillium pinophilum/verruculosum & $\mathrm{MX}-\mathrm{C} 1$ & biocontrol product & Mexico & ITS \\
\hline Penicillium polonicum & D_D47 & Indoor air & Austria & ITS-LSU \\
\hline Penicillium restrictum & PRF-18 & Ectomycorrhizal Salix root tip & Austria; Arnoldstein & ITS-LSU \\
\hline Penicillium roseopurpureum-related & D_D20 & Indoor air & Austria & ITS-LSU \\
\hline Penicillium rugulosum & ÖFI 05-01F & & Austria; Vienna & ITS \\
\hline Penicillium soppi & RSF-Q201 & Root sample Quercus & Austria; Redlschlag & ITS \\
\hline Penicillium spinulosum & Li0102XIX & Spruce needle & Austria; Kalwang & ITS-LSU \\
\hline Penicillium ochrochloron & NG_25 & Grassland soil & Austria; Riederberg & ITS \\
\hline
\end{tabular}


TABle 1: Continued.

\begin{tabular}{|c|c|c|c|c|}
\hline Species & Internal strain code & Isolation source & Country & Sequencing $^{\circ}$ \\
\hline Phanerochaete chrysosporium & P.c. & & & ITS \\
\hline Rhizopus oryzae & R.R. & Rapeseed pellets & & ITS \\
\hline Trichoderma atroviride & GAbT & Indoor air & Germany & ITS \\
\hline Trichoderma tomentosum & NG_02 & Agricultural soil & Austria; Niederschleinz & ITS \\
\hline Trichoderma viride & NG_p24 & Grassland soil & Austria; Riederberg & ITS \\
\hline
\end{tabular}

${ }^{\circ}$ Identification of the fungi was based on sequencing of the fungal internal transcribed spacer (ITS) region and partial LSU regions (ITS/LSU) according to Klaubauf et al. [28].

mechanically using a Fast Prep FP120 (three times for $30 \mathrm{sec}$ ) and centrifugated two times at $5500 \mathrm{rpm}$ for $10 \mathrm{~min}$ at $4^{\circ} \mathrm{C}$ and $800 \mu \mathrm{L}$ of the supernatant was filtered through a syringe filter (Rotilabo Syringe filter $\emptyset 0.22 \mu \mathrm{m}$; Roth, Germany). For the preparation of the extracted pellet, $1 \mathrm{~g}$ of the mycelium was mixed with $1 \mathrm{~mL}$ of chloroform and glass beads according to the manufacturer's recommendation. The cells were lysed as described above and $600 \mu \mathrm{L}$ of the supernatant was transferred into a new tube. The supernatant was further evaporated in a centrifugal vacuum concentrator at $30^{\circ} \mathrm{C}$ and resuspended in Moser medium supplemented with 10\% DMSO.

2.4. Antimicrobial Activity Screen. For the setup of the initial antimicrobial activity screen, we investigated the growth of $S$. aureus, C. albicans, and $P$. aeruginosa at $37^{\circ} \mathrm{C}$ for $48 \mathrm{~h}$ in four cultivation media: Luria-Bertani (LB, Oxoid, USA), tryptone soya broth supplemented with yeast extract (TSBY), Winge $(20 \mathrm{~g} / \mathrm{L}$ glucose, $3 \mathrm{~g} / \mathrm{L}$ yeast extract), and yeast extracted peptone dextrose (YEPD, Oxoid, USA) using glass tubes and 96-well microtiter plates. TBSY was chosen as cultivation medium. After three cultivations for $18 \mathrm{~h}$ in TSBY at $37^{\circ} \mathrm{C}$, cultures were diluted to an $\mathrm{OD}_{600}$ of 0.05 for $S$. aureus and of 0.5 for $C$. albicans and $P$. aeruginosa. The initial screen was performed in 96-well flat bottom microtiter plates (Greiner bio-one, Austria). Per well, $80 \mu \mathrm{L}$ of fresh TSBY was mixed with $100 \mu \mathrm{L}$ of fungal extract (supernatant, extracted supernatant, pellet, and extracted pellet) and additionally $20 \mu \mathrm{L}$ of the test organism culture $\left(\mathrm{OD}_{600}\right.$ of 0.05 for $S$. aureus, 0.5 for $P$. aeruginosa, and C. albicans) was added to reach the final reaction volume of $200 \mu \mathrm{L}$. As controls, Moser medium alone, with the specific SCs with 10\% DMSO, was used. The growth was measured after 0,6 , and $18 \mathrm{~h}$ of incubation at $37^{\circ} \mathrm{C}$ using a multiplate photometric reader (Tecan Infinite 200pro, Switzerland). Every experiment was performed in three biological replicates in triplicate. To validate the screening method, the intra- and interassay coefficients of variation (CV) were calculated according to Reed et al. [29].

For the enhanced antimicrobial activity screen, E. dermatitidis was cultivated three times for three days at $37^{\circ} \mathrm{C}$, diluted to an $\mathrm{OD}_{600}$ of 0.5 , and incubated in TSBY in glass tubes for $54 \mathrm{~h}$ at $37^{\circ} \mathrm{C}$. $\mathrm{OD}_{600}$ measurements were performed at $0,6,18,36$, and $54 \mathrm{~h}$ due to the slow growth rate. In addition, Klebsiella pneumonia, E. coli, ESBL K. pneumonia, ESBL E. coli, and the MRSA strain were cultivated for 18 hours in TSBY at $37^{\circ} \mathrm{C}$. Growth experiments were performed with a start inoculum of 0.05 in glass tubes at a final volume of $2 \mathrm{~mL}$ and $\mathrm{OD}_{600}$ was measured in a spectrophotometer (UV1800, Shimadzu, Japan).

2.5. Evaluation of Antimicrobial Activity of Fungal Cultures. The growth of the test organisms in the presence of the SC treated fungal samples was compared to the growth in the presence of the untreated fungal samples and to the growth of the test organisms alone. In addition, growth in the presence of the different SCs and DMSO was used as a control.

Of all fungal samples displaying antimicrobial activity, a colony forming unit assay (CFU) was performed. The determination of CFU was performed simultaneously with the $\mathrm{OD}_{600}$ measurements by serial dilution plating on tryptone soya agar plates, and the CFUs were evaluated $24 \mathrm{~h}$ after incubation at $37^{\circ} \mathrm{C}$. The CFU assay was done in three independent biological replicates performed in triplicate. Controls were used similar to the $\mathrm{OD}_{600}$ measurement.

2.6. Cytotoxicity Assay. A cytotoxicity assay measuring lactate dehydrogenase (LDH) release was performed using human intestinal epithelial (Caco2) and human hepatocytic (HepG2) cells. The cells were cultivated in Eagle's minimum essential medium (MEM) containing $2 \mathrm{mM} \mathrm{L}$-glutamine, 10\% fetal bovine serum (FBS), 100 units/mL penicillin, $100 \mathrm{mg} / \mathrm{mL}$ streptomycin sulfate, $0.25 \mathrm{mg} / \mathrm{mL}$ amphotericin $\mathrm{B}$, and $1 \%$ nonessential amino acids (NEAA; all PAA) at $37^{\circ} \mathrm{C}$ in a humidified atmosphere (95\% relative humidity) containing $5 \% \mathrm{CO}_{2}$.

The cells were seeded in 96 -well plates $\left(5 \times 10^{4}\right.$ per well $)$ and incubated until a confluent cell layer developed. For the cytotoxicity assay, cells were treated with $50 \mu \mathrm{L}$ fungal fraction and $50 \mu \mathrm{L}$ MEM for $24 \mathrm{~h}$ at $37^{\circ} \mathrm{C}$ and $\mathrm{LDH}$ leakage was determined using the $\mathrm{LDH}$ in vitro toxicology assay kit according to the manufactures' instructions (Sigma-Aldrich, USA). The percentage of dead cells was calculated using a standard curve of serial diluted lysed cells (100\% dead cells). Each experiment was performed three times in triplicate.

2.7. Statistical Analysis. Microsoft Excel 2007 and Sigma plot 11.0 (Sysat Software Inc., Great Britain) were used for statistical analysis. Values were compared statistically using $t$-test and Dunnett test (two independent variables). $P$ values $<0.05$ were considered to be significant. 
TABLE 2: Intra- and inter-assay coefficient of variation $(\mathrm{CV})$ of biological and technical replicates.

\begin{tabular}{lcccc}
\hline & \multicolumn{2}{c}{ Inter-assay CV [\%] } & \multicolumn{2}{c}{ Intra-assay CV [\%] } \\
& $6 \mathrm{~h}$ & $18 \mathrm{~h}$ & $6 \mathrm{~h}$ & $18 \mathrm{~h}$ \\
\hline S. aureus & 3.1 & 5.8 & 2.1 & 2.9 \\
P. aeruginosa & 4.9 & 6.5 & 2.0 & 4.3 \\
C. albicans & 9.4 & 24.4 & 7.6 & 17.4 \\
\hline
\end{tabular}

\section{Results}

3.1. Development of the Antimicrobial Activity Screen. For the setup of the antimicrobial activity screen, we tested four liquid media (LB, TSBY, Winge, and YEPD) commonly used in microbiology (see Table S1 in Supplementary Material available online at http://dx.doi.org/10.1155/2014/540292). TSBY showed the smallest difference in growth dynamics and best growth rate for all three test organisms in both the glass tube and 96-well microtiter plate assay (Table S1). In addition, we tested different inoculum sizes $\left(\mathrm{OD}_{600} 0.001-\right.$ 0.1 ) and revealed the lowest differences in growth dynamics using an inoculum size of $\mathrm{OD}_{600} 0.005$ for $S$. aureus and of $\mathrm{OD}_{600} 0.05$ for $P$. aeruginosa and $C$. albicans. Additional, by using these inoculum sizes, all three test organisms were in a comparable growth phase after 6 and $18 \mathrm{~h}$ of incubation at $37^{\circ} \mathrm{C}$, after $6 \mathrm{~h}$ in the mid- or end logarithmic, and after $18 \mathrm{~h}$ in the stationary growth phase (Figure S1). In parallel, CFUs were determined after 6 and $18 \mathrm{~h}$ to confirm the growth dynamics (data not shown). For the antimicrobial activity screen, the test organisms were grown at a ratio 1 to 1 in TSBY and the fungal samples were resuspended in Moser medium with and without DMSO. Moser medium did not influence the growth dynamics of all three test organisms. Furthermore, neither one of the SCs at the given concentration nor $2.5 \%$ DMSO had an effect on the growth of all three test organisms (data not shown).

3.2. Validation of the Antimicrobial Activity Screen. To validate the variability and reproducibility of the bioactivity screens, we calculated the intra- and interassay coefficients of variation $(\mathrm{CV})$. The intra-assay $\mathrm{CV}$ describes the reproducibility of technical replicates; interassay CV specifies the reproducibility of biological replicates [29]. Overall, the intraassay CV is lower than the interassay CV (Table 2). The variability of growth of both assays is below $10 \%$ for S. aureus and $P$. aeruginosa at 6 and $18 \mathrm{~h}$ and for C. albicans at $6 \mathrm{~h}$. The highest inter- and intra-assay CV was determined after $18 \mathrm{~h}$ for C. albicans; therefore, growth of C. albicans was only evaluated at the time point of $6 \mathrm{~h}$, respectively.

\subsection{Influence of the Maturation of Spores on the Antimicrobial} Activity. Visible sporulation of all fungi took place after 8 days of incubation at RT. We observed that the age of the spores used for the fungal cultures influenced the production of antimicrobial compounds. For instance, the untreated supernatant of Oidiodendron (O.) cerealis (NG_p39) showed a significantly higher antimicrobial activity against $S$. aureus, if we inoculated 10- and 20-day-old spores, whereas no antimicrobial activity could be observed by using 8 -day-old spores (Figure S2). Since no difference was observed between spores grown for either 10 or 20 days, we decided to harvest all spores of all fungi after 10 days of incubation.

3.4. SCs Induce Antimicrobial Activity in Fungi. In total, we investigated antimicrobial activity of fungal samples of 54 strains belonging to 11 different genera, which were grown in the presence of six different SCs. To include both intra- and extracellular antimicrobial compounds and the whole range of polar and apolar compounds, we prepared four fractions of each fungal culture: the supernatant and the mycelium, both crude and extracted. In total, we determined the effect of 1512 different fungal fractions on the growth of three different test organisms.

Thirty fungal samples of treated fungi belonging to six different genera reduced significantly growth of the test organism compared to the untreated fungal sample (Table 3). Of these 30 samples, only two, namely, the supernatant of A. clavatus (L19) and $H$. koningii (NG_14 BRE1P1), showed already antimicrobial activity in the untreated sample, although to a lower extent compared to the treated fraction.

All six SCs induced the production of antimicrobial compounds at least in the fraction of one fungus; additional increased bioactivity was observed in all four different fungal fractions, respectively. In total, 12 fungal samples showed activity against $C$. albicans, 10 against $S$. aureus, and 8 against $P$. aeruginosa. Overall, we observed cell count reductions varying between 0.3 and 4.3 (Table 3 ).

The most salient growth suppressing effects were as follows: we detected a strong antimicrobial activity against all three test organisms in the supernatant of $A$. clavatus (L19). Although antimicrobial activity was already detected in the supernatant of the untreated fungus, treatment with VPA, TSA, butyrate, AZA, and GlcNAc resulted in a significantly increased bioactivity. The highest antimicrobial activity, however, was observed in the supernatant of $A$. clavatus (L19) grown in the presence of AZA, resulting in a reduction of $S$. aureus numbers of $4.3 \log \mathrm{CFU}$ (Figures 1(a) and 1(b)). In addition, these supernatants inhibited also growth of E. dermatitidis, Klebsiella pneumonia, E. coli, ESBL K. pneumonia, ESBL E. coli, and MRSA strain (data not shown).

Increased antimicrobial activity against $P$. aeruginosa, resulting in a CFU log reduction of 4 , was measured also in the supernatant of $H$. koningii (NG_14 BRE-1P1) grown in the presence of $\mathrm{NO}$ compared to untreated supernatant (Figures $1(\mathrm{c})$ and $1(\mathrm{~d})$ ). However, in this fungus, bioactivity was already detected in the supernatant of the untreated fungi.

The pellet of $P$. restrictum (PRF-18) grown in the presence of butyrate showed antimicrobial activity against $S$. aureus compared to the untreated fungal sample (and the control), even though the growth of the test organisms was increased in the presence of the untreated pellet (Figure 2(a)). In parallel, this fungal fraction revealed a $1.4 \log$ reduction in $S$. aureus numbers (Figure 2(b)). In the enhanced screen, this 
TABLE 3: Fungal samples showing increased antimicrobial activity after SC treatment compared to the untreated fungal sample.

\begin{tabular}{|c|c|c|c|c|}
\hline Fungal strain & Fungal fraction & SC & Target & Log reduction $^{\circ}$ \\
\hline \multirow{15}{*}{ Aspergillus clavatus* } & \multirow{15}{*}{ Supernatant } & AZA & \multirow{5}{*}{ S. aureus } & $4.28 \pm 0.745$ \\
\hline & & Butyrate & & $3.01 \pm 0.521$ \\
\hline & & TSA & & $3.89 \pm 0.637$ \\
\hline & & VPA & & $3.75 \pm 0.419$ \\
\hline & & GlcNAc & & $0.72 \pm 0.187$ \\
\hline & & AZA & \multirow{5}{*}{ P. aeruginosa } & $4.03 \pm 0.923$ \\
\hline & & Butyrate & & $3.24 \pm 0.427$ \\
\hline & & TSA & & $4.12 \pm 0.845$ \\
\hline & & VPA & & $4.04 \pm 0.736$ \\
\hline & & GlcNAc & & $1.25 \pm 0.249$ \\
\hline & & AZA & \multirow{5}{*}{ C. albicans } & $2.01 \pm 0.423$ \\
\hline & & Butyrate & & $1.84 \pm 0.359$ \\
\hline & & TSA & & $2.04 \pm 0.476$ \\
\hline & & VPA & & $3.12 \pm 0.863$ \\
\hline & & GlcNAc & & $1.36 \pm 0.419$ \\
\hline Clavicipitaceae spp. & Extracted supernatant & Butyrate & C. albicans & $1.10 \pm 0.301$ \\
\hline Hypocrea koningii ${ }^{*}$ & Supernatant & $\mathrm{NO}$ & P. aeruginosa & $4.00 \pm 0.673$ \\
\hline Isaria farinosa & Extracted supernatant & Butyrate & C. albicans & $0.37 \pm 0.084$ \\
\hline \multirow{3}{*}{ Penicillium alberechii } & \multirow{3}{*}{ Supernatant } & TSA & \multirow{3}{*}{ S. aureus } & $0.47 \pm 0.035$ \\
\hline & & Butyrate & & $0.51 \pm 0.074$ \\
\hline & & VPA & & $0.49 \pm 0.063$ \\
\hline Penicillium crustosum & Extracted pellet & TSA & C. albicans & $1.10 \pm 0.451$ \\
\hline \multirow{3}{*}{ Penicillium decaturense } & \multirow{3}{*}{ Supernatant } & AZA & \multirow{3}{*}{ C. albicans } & $0.35 \pm 0.032$ \\
\hline & & Butyrate & & $0.30 \pm 0.012$ \\
\hline & & TSA & & $0.31 \pm 0.057$ \\
\hline Penicillium miczynskii & Extracted supernatant & $\mathrm{NO}$ & C. albicans & $0.74 \pm 0.087$ \\
\hline Penicillium restrictum & Pellet & Butyrate & S. aureus & $1.40 \pm 0.204$ \\
\hline Penicillium soppi & Extracted supernatant & TSA & P. aeruginosa & $0.56 \pm 0.096$ \\
\hline Penicillium spinulosum & Supernatant & GlcNAc & S. aureus & $0.94 \pm 0.061$ \\
\hline Trichoderma atroviride & Pellet & AZA & P. aeruginosa & $0.42 \pm 0.085$ \\
\hline
\end{tabular}

${ }^{\circ} \mathrm{CFU} \log$ reduction comparing treated with untreated fungal fractions determined after 6 hours of growth at $37^{\circ} \mathrm{C}$. Data are represented as mean values \pm standard deviations of three biological replicates preformed in triplicate. ${ }^{*}$ Untreated fungal fraction showed already antimicrobial activity.

P. restrictum (PRF-18) sample showed antimicrobial activity against the MRSA strain (Figure 2(c)).

With regard to $P$. crustosum (D_D27), TSA increased the antimicrobial activity against $C$. albicans in the extracted pellet (Figure 3(a)). In parallel, the number of viable C. albicans cells decreased, (CFU log reduction of 1.1, Figure 3(b)) and the antimicrobial effect of this fungal sample on the growth of $E$. dermatitidis, a slow growing fungus, was even stronger compared to the effect on C. albicans (Figure 3(c)).

P. spinulosum (Li0102XIX) was analytically challenging, since the supernatant displayed weak turbidity leading to higher $\mathrm{OD}_{600}$ at the start point $(0 \mathrm{~h})$. The presence of GlcNAc increased antimicrobial activity against $S$. aureus in the supernatant of this organism (Figure 4). We observed that the untreated crude fungal supernatant already increased the growth S. aureus compared to the control.

3.5. Cytotoxicity of Fungal Samples. To get a first insight into the host susceptibility, we investigated the cytotoxicity of the respective fungal fraction of $A$. clavatus (L19), $H$. koningii (NG_14 BRE-1P1), P. restrictum (PRF-18), and P. crustosum (D_D27) using two human cell lines (Caco2 and HepG2,
Figure 5). The cytotoxic effects of the fungal samples were strain and cell line dependent (\% of dead cells: $0-42 \%$ ).

The incubation of the cells with Moser medium alone resulted in 3.6 to $5 \%$ dead cells. All but one tested fractions of fungi treated with the respective SC showed higher cytotoxicity compared to the untreated crude fraction. Only the pellet of $P$. restrictum (PRF-18) grown in the presence of butyrate revealed fewer dead $\mathrm{Caco} 2$ cells. Overall, both fractions of P. restrictum (PRF-18) were not cytotoxic, revealing $0-2.8 \%$ dead cells after $24 \mathrm{~h}$ of incubation, whereas the treated fungal fractions of $H$. koningii (NG_14 BRE-1P1) and P. crustosum (D_D27) were highly cytotoxic, resulting in $40 \%$ dead cells. The treated fraction of A. clavatus (L19) displayed moderate cytotoxicity ranging from $8 \%$ dead cells for $\mathrm{Caco} 2$ to $21 \%$ for HepG2 cells.

\section{Discussion}

The increasing need for novel antimicrobial drugs raises the necessity to establish alternative sources and highthroughput methods for antimicrobial compound discovery. 

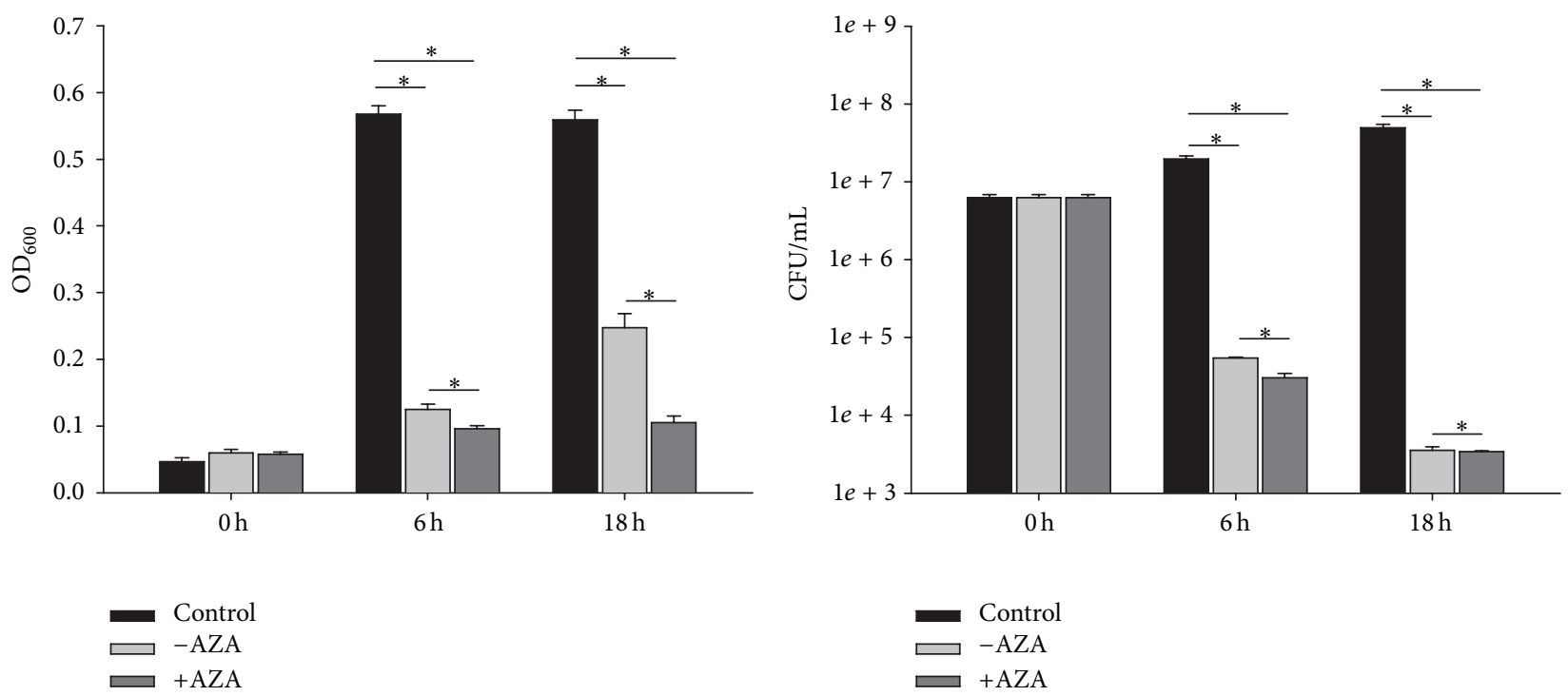

(a)
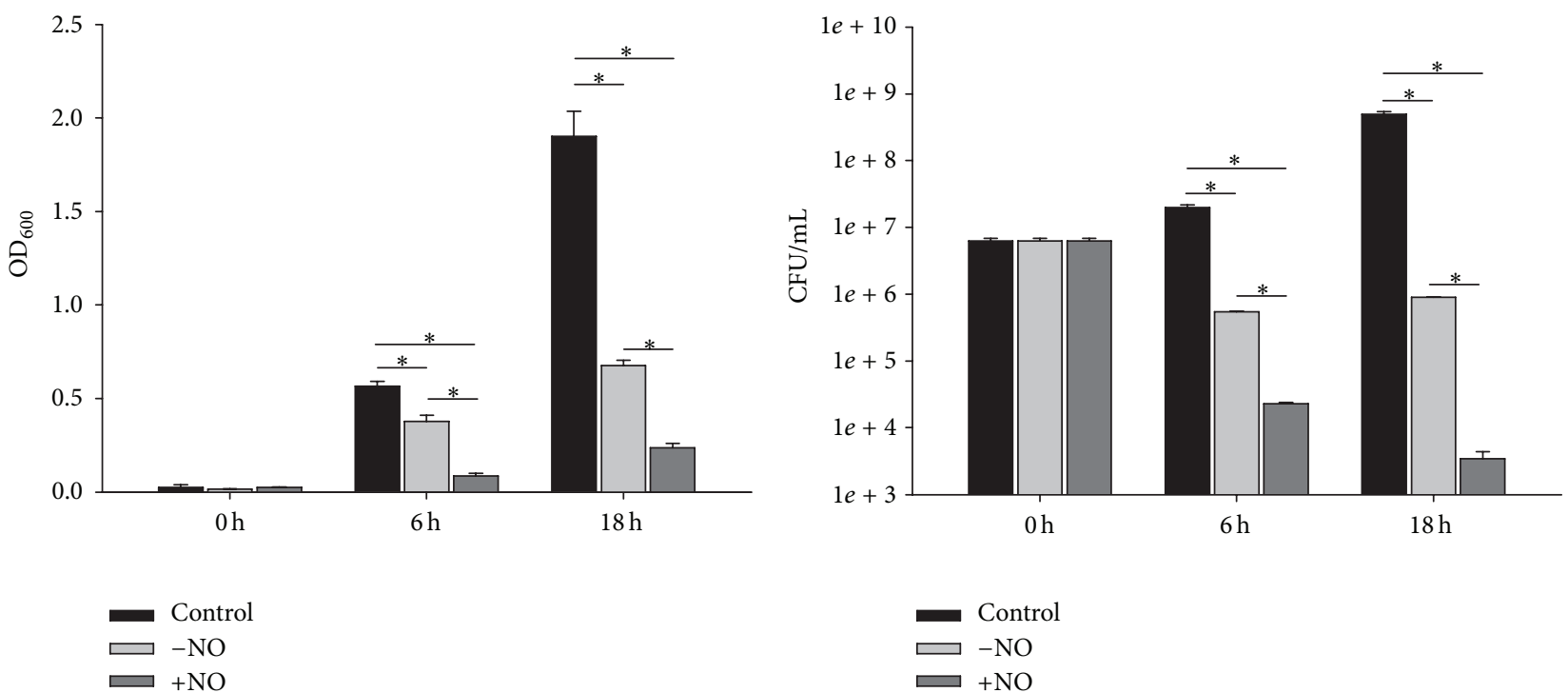

(c)

(d)

Figure 1: Antimicrobial effects of the AZA treated (+AZA) and untreated (-AZA) supernatant of A. clavatus (L19) on the growth of S. aureus ((a): $\mathrm{OD}_{600}$, (b): $\left.\mathrm{CFU} / \mathrm{mL}\right)$. Antimicrobial effects of the $\mathrm{NO}$ treated (+NO) and untreated (-NO) supernatant of H. koningii (NG_14 BRE$1 \mathrm{P1}$ ) on the growth of $P$. aeruginosa ((c): $\left.\mathrm{OD}_{600},(\mathrm{~d}): \mathrm{CFU} / \mathrm{mL}\right)$. Control comprises S.aureus or P. aeruginosa grown without fungal sample. Data is presented as mean values \pm standard deviations of three biological replicates performed in triplicate. ${ }^{*}$ indicates significant difference $(P<0.05)$.

Recent progress in fungal sequencing and in the understanding of epigenetic regulation mechanisms suggests a large number of so far unknown secondary metabolites which may include antimicrobial active compounds $[4,6]$. In this study, we describe and evaluate a strategy to discover new natural antimicrobial compounds combining a yet unexploited approach using small mass chemicals to modify the secondary metabolite production in fungi with a sensitive antimicrobial activity screen.

The presented screening method was designed to determine the growth of three test organisms, which are known to acquire antibiotic resistance. These species are therefore commonly used to assess antimicrobial activity. The growth medium TSBY, an unselective growth medium, is suited for receiving low variability with respect to growth dynamics. Therefore, biased positive results due to nutrient limitation, selective pressure, or medium variability are negligible. When validating the antimicrobial screens, the variability and reproducibility revealed remarkable low inter- and intraassay CVs, which indicates low media batch variation and an excellent opportunity to detect also weak antimicrobial activity. Both CVs were below $10 \%$ for all test organisms after 

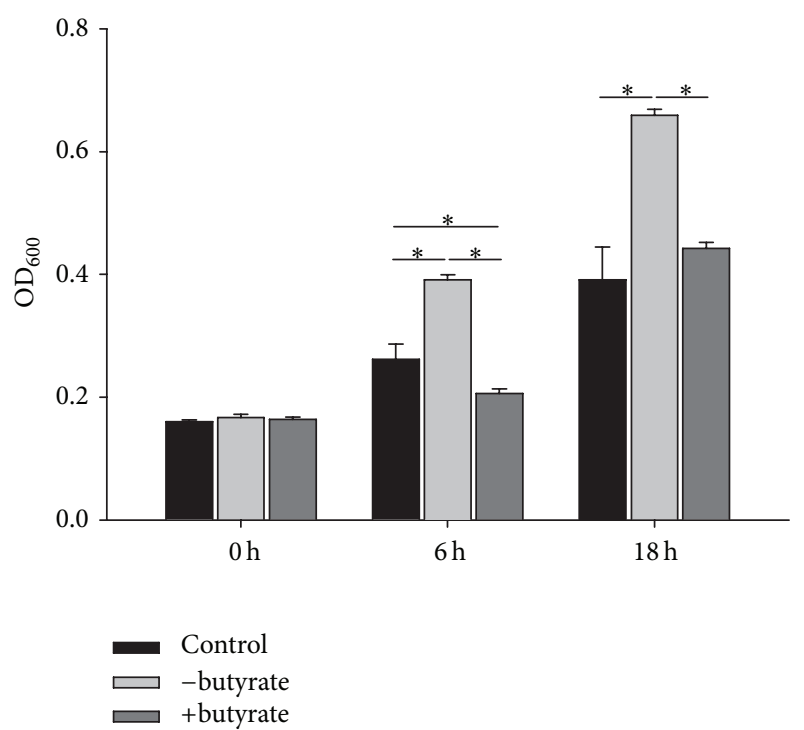

(a)
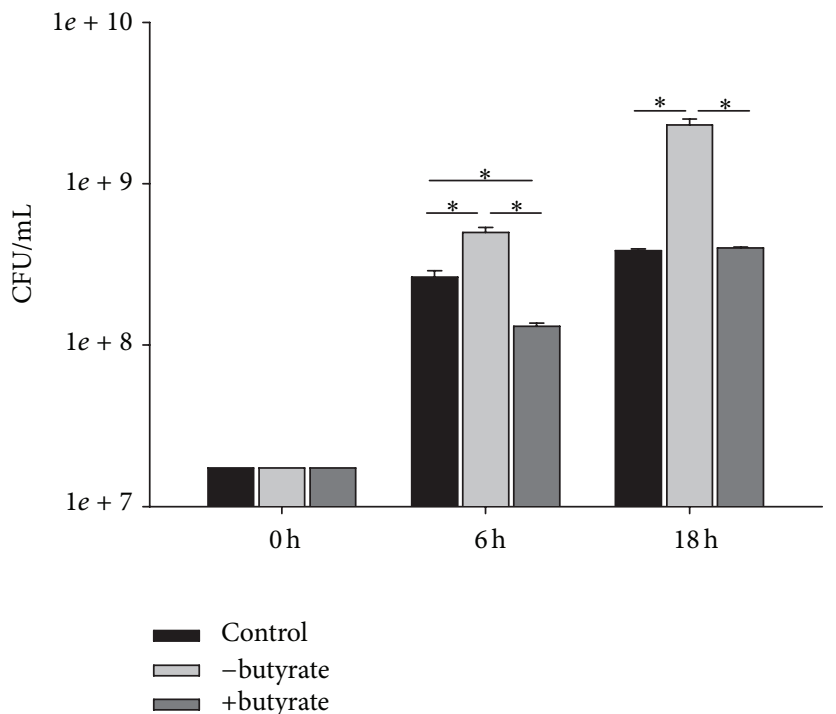

(b)

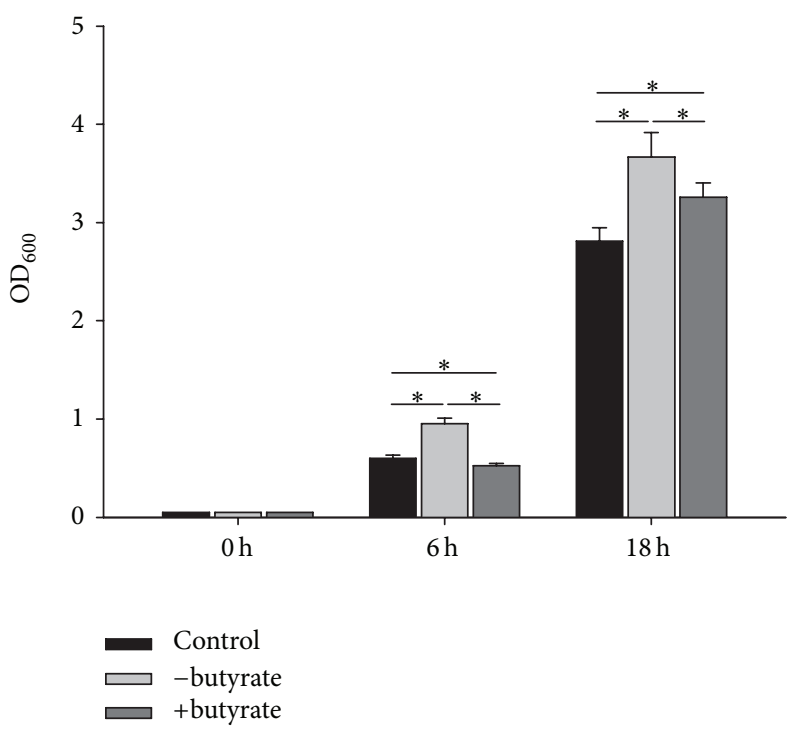

(c)

FIGURE 2: Antimicrobial activity of the pellet of P. restrictum (PRF-18) grown in the absence (-butyrate) and presence (+butyrate) of butyrate against $S$. aureus ((a): $\left.\mathrm{OD}_{600},(\mathrm{~b}): \mathrm{CFU} / \mathrm{mL}\right)$ and the MRSA strain $\mathrm{B} 337919\left((\mathrm{c}): \mathrm{OD}_{600}\right)$. Control comprises $S$. aureus or MRSA grown without fungal samples. Data is represented as mean values \pm standard deviations of three biological replicates performed in triplicate. ${ }^{*}$ indicates significant difference $(P<0.05)$.

6 and $18 \mathrm{~h}$ of growth. Comparable studies using screening systems for bacterial susceptibility against common antibiotics reported inter-assay CV values of $10-20 \%$ depending on the test organism [30]. The only exception was the growth variability of C. albicans when incubated for 18 h. C. albicans clusters at the bottom of the reaction well resulting in a poorer resuspension before OD measurements.

In total, 54 fungi including 12 Aspergilli and 30 Penicillia strains were treated with six SCs and screened for novel antimicrobial compounds in four fractions. All fungi were grown in the same medium and kept under constant conditions to avoid influence of biotic and abiotic factors on the expression of secondary metabolites. The high number of
Aspergilli and Penicillia strains was chosen due to the large amount of genomic, proteomic, and ecological data available for these genera. In addition, recent studies showed that even closely related fungi share only a small number of similar secondary metabolites and that horizontal gene transfer of biosynthesis clusters of secondary metabolites is a scarce event $[31,32]$.

We detected 30 samples of treated fungi belonging to six different genera, with seven Penicillia strains among them, which reduced significantly growth of the test organism compared to the untreated fungal samples. All SCs used in this study effected the production of antimicrobial compounds at least in one fungus. However, whether the effect 


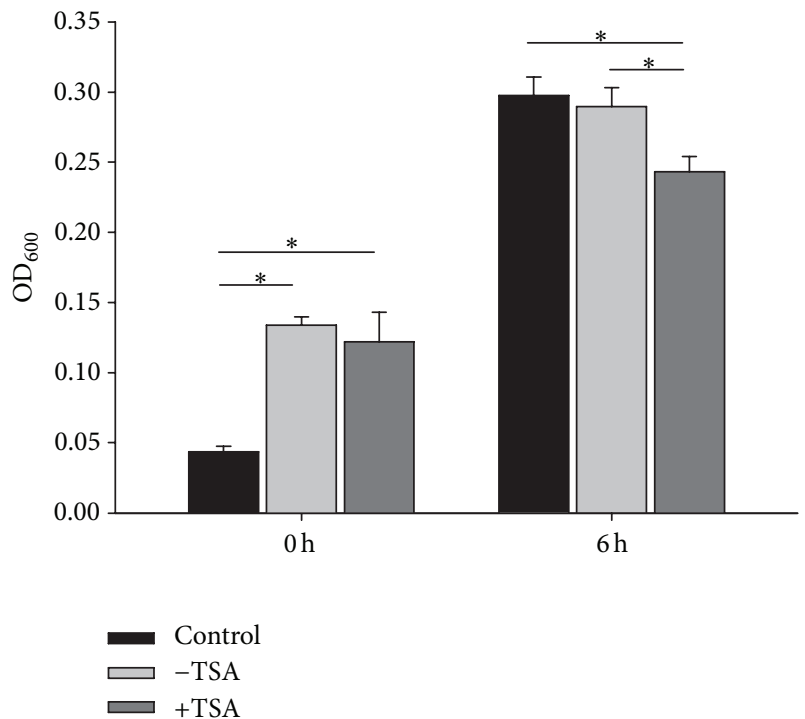

(a)
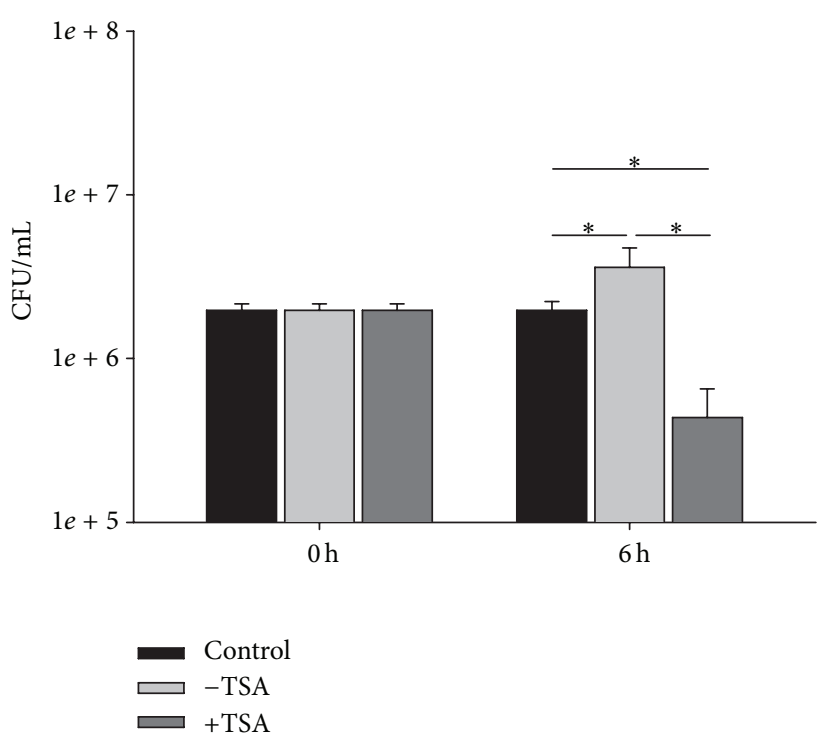

(b)

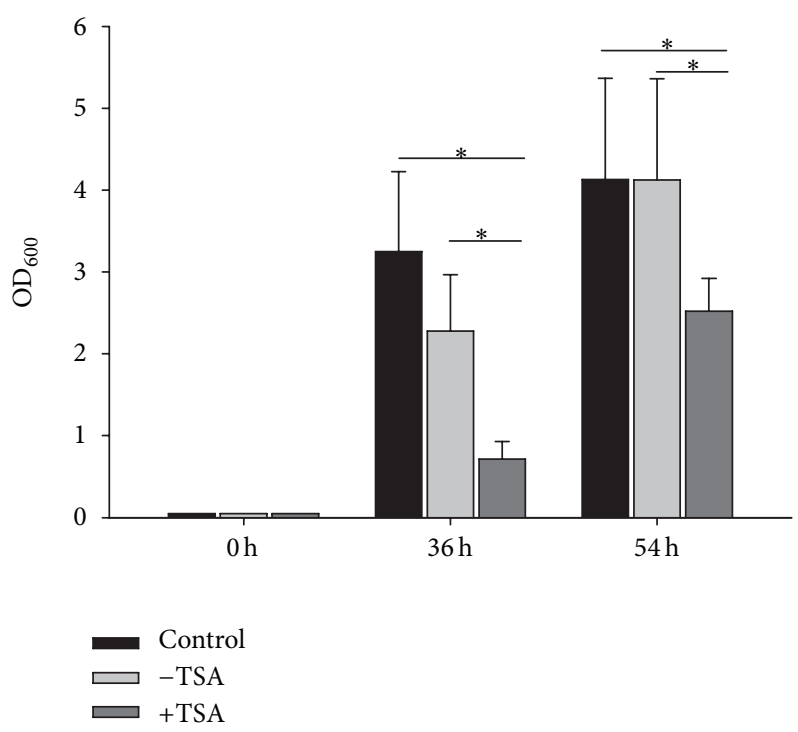

(c)

FIGURE 3: Antimicrobial activity against C. albicans ((a): $\left.\mathrm{OD}_{600},(\mathrm{~b}): \mathrm{CFU} / \mathrm{mL}\right)$ and E. dermatitidis ((c): $\left.\mathrm{OD}_{600}\right)$ in the extracted pellet of P. crustosum (D_D27) grown with (+TSA) and without TSA (-TSA). Controls comprise C. albicans and E. dermatitidis grown without fungal sample. Data comprises mean values \pm standard deviations of three biological replicates performed in triplicate. ${ }^{*}$ indicates significant difference $(P<0.05)$.

of the SC on the production of antimicrobial compounds is due to epigenetic modulation in the specific fungus must still be investigated. Interestingly, except for $A$. clavatus, increased antimicrobial activity was only detected in one of the four fungal fractions of all fungi. Furthermore, growth was inhibited either of yeast, gram positive, or gram negative bacteria (except $A$. clavatus). This is in line with recent findings that SCs are target specific, inducing specific secondary metabolite production $[16,33]$.

Excluding A. clavatus, seven of these modified fungal fractions showed activity against yeast, five against gram positive bacteria, and only three against gram negative bacteria. The small number of fungal fractions displaying activity against gram negative bacteria underlines the poor availability of antibiotics against gram negative bacteria and underlines that few novel candidates are in the pipeline [34].

In the supernatant of untreated and treated A. clavatus, we revealed high antimicrobial activity against all tested organisms and cytotoxicity. A. clavatus is known to produce the mycotoxin patulin, which is cytotoxic and highly bioactive also against gram positive and negative bacteria [35, 36]. Both the antimicrobial activity and cytotoxicity were increased if this fungus was grown in the presence of all SCs. These findings are in line with one of our studies, which shows that the SCs increased, among other mycotoxins, patulin production in A. clavatus [16]. 

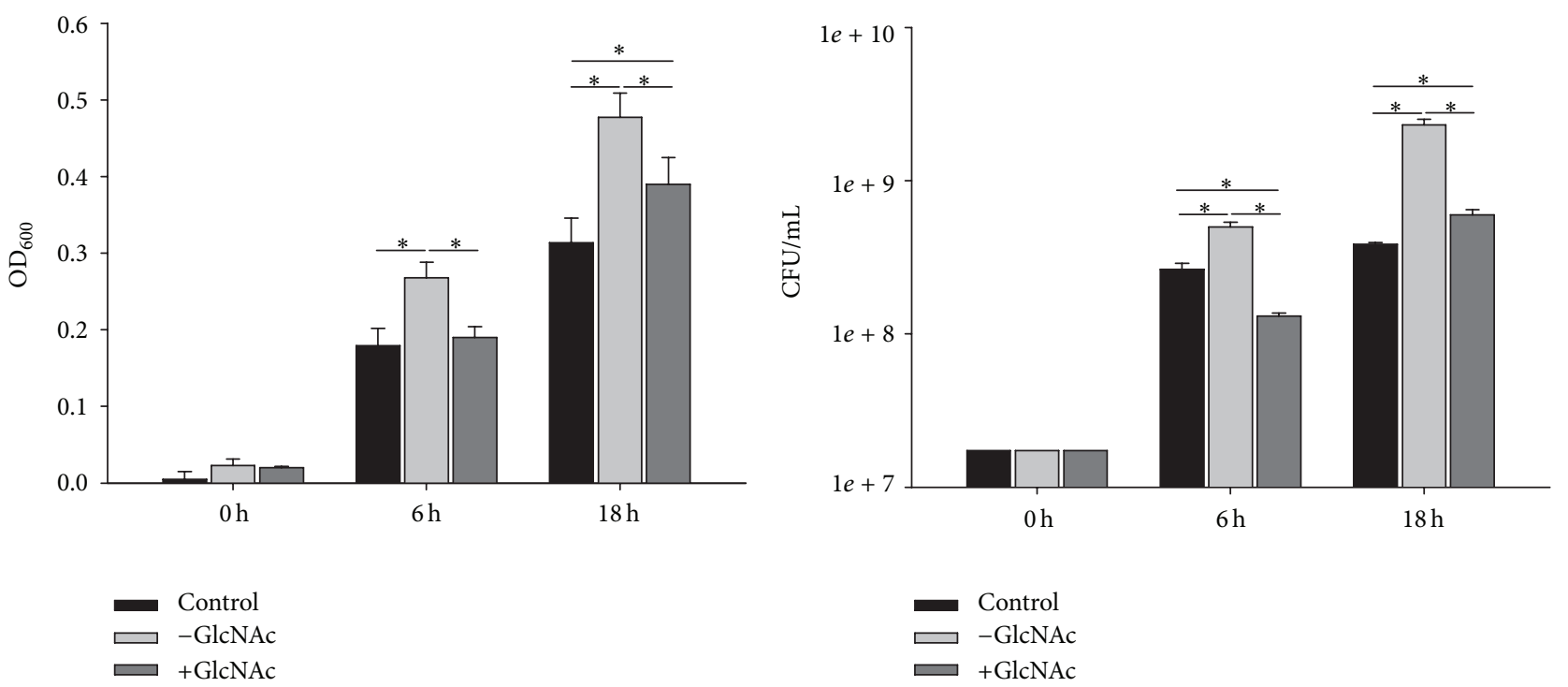

(a)

(b)

Figure 4: Effect of the GlcNAc treated (+GlcNAc) and untreated (-GlcNAc) supernatant of P. spinulosum (Li0102XIX) on the growth of S. aureus measured by $\mathrm{OD}_{600}(\mathrm{a})$ and $\mathrm{CFU} / \mathrm{mL}$ (b). Control comprises $S$. aureus grown without fungal sample. Data are represented as mean values \pm standard deviations of three biological replicates performed in triplicate. ${ }^{*}$ indicates significant differences $(P<0.05)$.

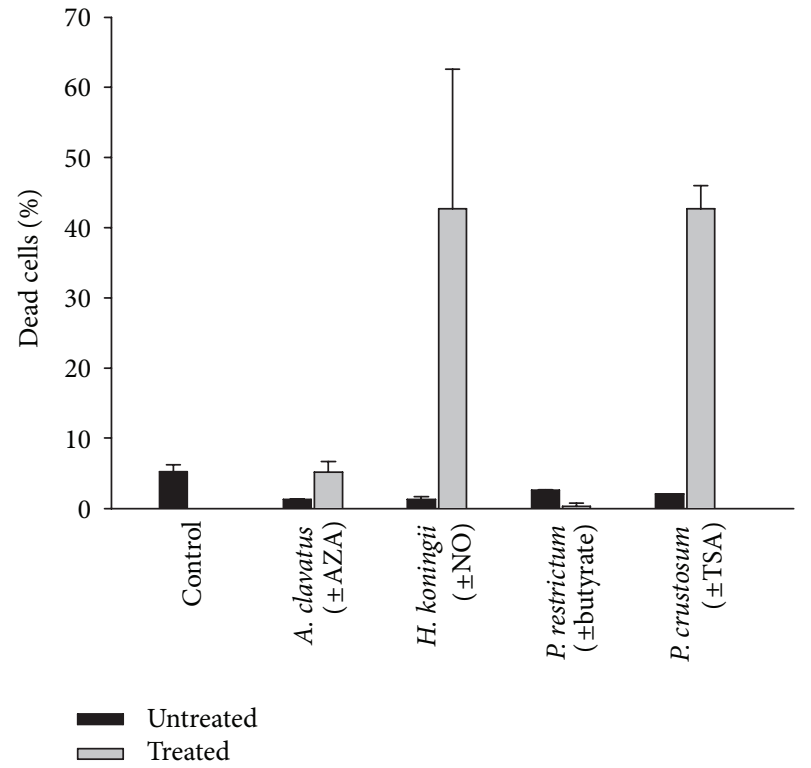

(a)

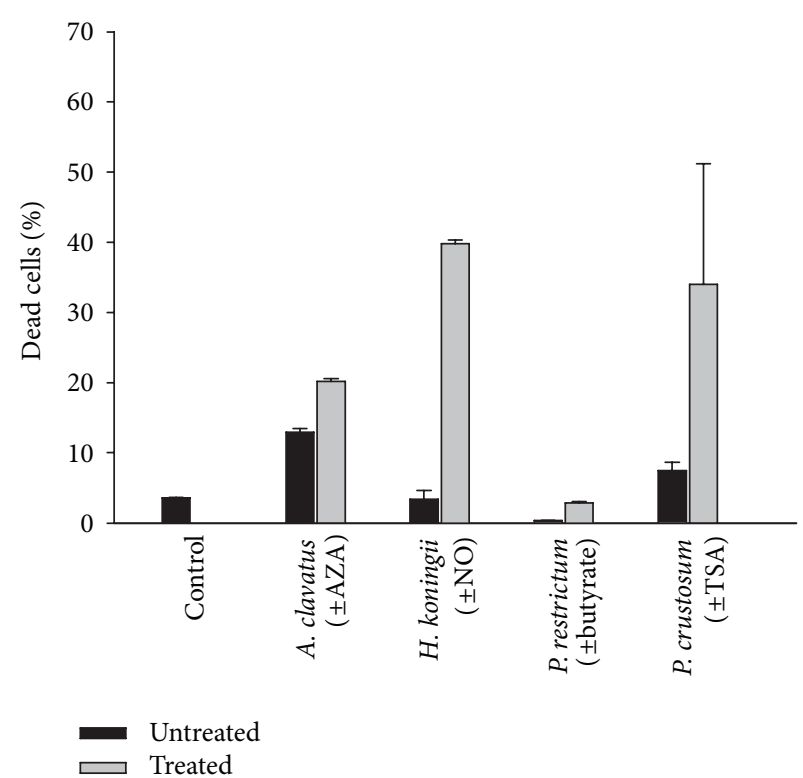

(b)

FIgure 5: Cytotoxic effects of the supernatant of $A$. clavatus (L19), the supernatant of $H$. koningii (NG_14 BRE-1P1), the pellet of P. restrictum (PRF-18), and the extracted pellet of P. crustosum (D_D27) incubated with (treated) and without (untreated) the respective SC using human intestinal epithelial Caco2 (a) and hepatocytic HepG2 (b) cells. Control comprises Moser medium. Values, given as \% of dead cells, represent mean values \pm standard deviations of three biological replicates performed in triplicate.

In addition, the crude supernatant of $H$. koningii revealed already high antimicrobial activity against $P$. aeruginosa; however, the presence of NO resulted in increased bioactivity and cytotoxicity. It has been described that $H$. koningii produces various koninginins, which display a wide range of bioactivities including antimicrobial activity against gram negative bacteria $[37,38]$. Our observation that also the addition of NO increases the antimicrobial activity of $H$. koningii (and also in P. miczynskii) supports the hypothesis that the phytohormone $\mathrm{NO}$ influences $\mathrm{SM}$ production also in fungi, not only in plants $[26,27,39]$. At present, it is unknown if NO influences chromatin structure in fungi; therefore, further research is needed to study the role of NO in the regulation of secondary metabolite production. 
The finding that specific SCs increase the production of specific secondary metabolites like patulin and presumably koninginins highlights that small mass chemicals potentially inducing epigenetic modulation could be used for biotechnological approaches to increase the yield of specific compounds. Since A. clavatus and H. koningii showed antimicrobial activity already in the crude supernatant without any SCs, we disclosed these fractions from further analysis, because of the allegedly risk of rediscovery of known compounds.

Apart from A. clavatus and H. koningii, CFU log reductions between 0.3 and 1.4 were observed in the fungal samples, which corresponds to a relatively weak antimicrobial activity compared to the bacteriocidal effects of commercially available antimicrobial compounds. Optimization of biotic and abiotic factors, for example, temperature, light, $\mathrm{pH}$, nutrients, and oxygen availability, which are known to influence production of SM [40], could increase the yield of the antimicrobial compounds. Furthermore, there are indications that the age of the spores influences the production of antimicrobial compounds [41]. This is in line with our finding that, for example, in O. cerealis, which is known to produce the antimicrobial active compounds fuscin and clerocidin [42], antimicrobial bioactivity was only observed if the spores were grown for at least 10 days. Therefore, optimization of the age of spores could also increase the yield of antimicrobial compounds.

The observation that the pellet of $P$. restrictum grown in the presence of butyrate is not cytotoxic and revealed antimicrobial activity against $S$. aureus (CFU log reduction of 1.4) and MRSA indicates a high potential for the discovery of a novel antimicrobial compound in this fungal sample. No antimicrobial compounds active against gram positive bacteria have yet been described in any $P$. restrictum strain. It is known that $P$. restrictum produces calbistrin, which inhibits growth of fungi [43]. However, no antimicrobial activity against $C$. albicans has been observed in the fraction of the tested $P$. restrictum strain. Whether butyrate acts as an epigenetic modulator generally in fungi (including $P$. restrictum) is still unknown. So far, the epigenetic effect of butyrate via inhibition of HDACs (mainly of class II and III) has only been described in mammalian cells [22].

We observed that growth of $S$. aureus was increased in the presence of several fungal samples, both crude and treated, which indicates that fungal metabolites in both the pellet and the supernatant can be used by $S$. aureus as an additional nutrient source. In two of these cases (supernatant of GlcNac treated P. spinulosum and pellet of $P$. restrictum treated with butyrate), the presence of the SC treated fraction reduced significantly growth compared to the untreated fraction and also to the control, which suggests the presence of antimicrobial compounds. However, we cannot fully exclude that the specific SC also downregulates metabolites, which are used by $S$. aureus as nutrients. Taken together, the high number of antimicrobial active fungal samples in this study underlines the potential of this approach, combining treatment of fungi with small chemicals with a sensitive screening tool. In a common first screen of a set of 100 000-200 000 samples, $0.1-1 \%$ are estimated to be initial bioactive hits [44]. Up to $90 \%$ of these hits are predicted to be discarded because their bioactivity is a result of either a combination of weak bioactivities, due to biological variations or a dereplication of known compounds. This results in approximately 10-200 samples which are taken to further isolation and identification [44]. Our study comprising 1350 samples of 54 fungi detected 14 fungal samples containing potential novel antimicrobial compounds, which will be isolated and characterized in future studies. Compared to the estimated gain of 0.01$0.1 \%$ of an industrial screen, our method reached a gain of approximately $0.7 \%$. This indicates a large number of so far unknown antimicrobial compounds in already screened and characterized fungi which are accessible through to potential epigenetic regulation.

\section{Conclusion}

In conclusion, our study is the first to show the potential of SCs to induce the production of "cryptic" antimicrobial compounds against gram positive, gram negative bacteria, and yeasts. The diversity of the antimicrobial hits in the initial screen indicates that every presumable fungus, even well described fungi, has the potential to produce novel antimicrobial compounds that warrant discovery. In general, epigenetic modification of fungi could reduce the risk of rediscovery of known compounds. Furthermore, the used method combining treatment of fungi with small chemicals with a sensitive screening tool can easily be upscaled for highthroughput screening programs, discovering novel antimicrobial natural products.

\section{Conflict of Interests}

The authors declare that there is no conflict of interests regarding the publication of this paper.

\section{Acknowledgments}

The work was funded by the Vienna Science and Technology Fund "WWTF", Project "ToxiGenome" LS09-042.

\section{References}

[1] G. Molinari, "Natural products in drug discovery: present status and perspectives," Advances in Experimental Medicine and Biology, vol. 655, pp. 13-27, 2009.

[2] D. J. Newman and G. M. Cragg, "Natural products as sources of new drugs over the 30 years from 1981 to 2010," Journal of Natural Products, vol. 75, no. 3, pp. 311-335, 2012.

[3] P. W. Taylor, "Alternative natural sources for a new generation of antibacterial agents," International Journal of Antimicrobial Agents, vol. 42, no. 3, pp. 195-201, 2013.

[4] J. M. Winter, S. Behnken, and C. Hertweck, "Genomics-inspired discovery of natural products," Current Opinion in Chemical Biology, vol. 15, no. 1, pp. 22-31, 2011.

[5] C. T. Walsh and M. A. Fischbach, "Natural products version 2.0: connecting genes to molecules," Journal of the American Chemical Society, vol. 132, no. 8, pp. 2469-2493, 2010. 
[6] N. P. Keller, G. Turner, and J. W. Bennett, "Fungal secondary metabolism-from biochemistry to genomics," Nature Reviews Microbiology, vol. 3, no. 12, pp. 937-947, 2005.

[7] K. M. Fisch, A. F. Gillaspy, M. Gipson et al., "Chemical induction of silent biosynthetic pathway transcription in Aspergillus niger," Journal of Industrial Microbiology \& Biotechnology, vol. 36, no. 9, pp. 1199-1213, 2009.

[8] S. Bergmann, A. N. Funk, K. Scherlach et al., "Activation of a silent fungal polyketide biosynthesis pathway through regulatory cross talk with a cryptic nonribosomal peptide synthetase gene cluster," Applied and Environmental Microbiology, vol. 76, no. 24, pp. 8143-8149, 2010.

[9] Y. Chiang, C. E. Oakley, M. Ahuja et al., "An efficient system for heterologous expression of secondary metabolite genes in Aspergillus nidulans," Journal of the American Chemical Society, vol. 135, no. 20, pp. 7720-7731, 2013.

[10] H. W. Nützmann, Y. Reyes-Dominguez, K. Scherlach et al., "Bacteria-induced natural product formation in the fungus Aspergillus nidulans requires Saga/Ada-mediated histone acetylation," Proceedings of the National Academy of Sciences of the United States of America, vol. 108, no. 34, pp. 14282-14287, 2011.

[11] Y. Reyes-Dominguez, S. Boedi, M. Sulyok et al., "Heterochromatin influences the secondary metabolite profile in the plant pathogen Fusarium graminearum," Fungal Genetics and Biology, vol. 49, no. 1, pp. 39-47, 2012.

[12] A. Gacek and J. Strauss, "The chromatin code of fungal secondary metabolite gene clusters," Applied Microbiology and Biotechnology, vol. 95, no. 6, pp. 1389-1404, 2012.

[13] G. Brosch, P. Loidl, and S. Graessle, "Histone modifications and chromatin dynamics: a focus on filamentous fungi," The FEMS Microbiology Reviews, vol. 32, no. 3, pp. 409-439, 2008.

[14] P. A. Cole, "Chemical probes for histone-modifying enzymes," Nature Chemical Biology, vol. 4, no. 10, pp. 590-597, 2008.

[15] R. H. Cichewicz, "Epigenome manipulation as a pathway to new natural product scaffolds and their congeners.", Natural product reports, vol. 27, no. 1, pp. 11-22, 2010.

[16] C. Zutz, A. Gacek, M. Sulyok, M. Wagner, J. Strauss, and K. Rychli, "Small chemical chromatin effectors alter secondary metabolite production in Aspergillus clavatus," Toxins, vol. 5, no. 10, pp. 1723-1741, 2013.

[17] M. Göttlicher, S. Minucci, P. Zhu et al., "Valproic acid defines a novel class of HDAC inhibitors inducing differentiation of transformed cells," The EMBO Journal, vol. 20, no. 24, pp. 69696978, 2002.

[18] O. H. Krämer, P. Zhu, H. P. Ostendorff et al., "The histone deacetylase inhibitor valproic acid selectively induces proteasomal degradation of HDAC2," EMBO Journal, vol. 22, no. 13, pp. 3411-3420, 2003.

[19] W. L. Smith and T. D. Edlind, "Histone deacetylase inhibitors enhance Candida albicans sensitivity to azoles and related antifungals: correlation with reduction in CDR and ERG upregulation," Antimicrobial Agents and Chemotherapy, vol. 46, no. 11, pp. 3532-3539, 2002.

[20] E. P. Candido, R. Reeves, and J. R. Davie, "Sodium butyrate inhibits histone deacetylation in cultured cells," Cell, vol. 14, no. 1, pp. 105-113, 1978.

[21] J. Vrba, K. Trtkova, and J. Ulrichova, "HDAC inhibitors sodium butyrate and sodium valproate do not affect human ncorl and ncor2 gene expression in HL-60 cells," Biomedical Papers, vol. 155, no. 3, pp. 259-262, 2011.
[22] J. R. Davie, "Inhibition of histone deacetylase activity by butyrate," Journal of Nutrition, vol. 133, supplement 7, pp. 2485S2493S, 2003.

[23] E. L. Fritz and F. N. Papavasiliou, "Cytidine deaminases: AIDing DNA demethylation?” Genes \& Development, vol. 24, no. 19, pp. 2107-2114, 2010.

[24] S. Hagemann, O. Heil, F. Lyko, and B. Brueckner, "Azacytidine and decitabine induce gene-specific and non-random DNA demethylation in human cancer cell lines," PLOS ONE, vol. 6, no. 3, Article ID e17388, 2011.

[25] W. J. Chi, S. Y. Lee, and J. Lee, "Functional analysis of SGR4635induced enhancement of pigmented antibiotic production in Streptomyces lividans," Journal of Microbiology, vol. 49, no. 5, pp. 828-833, 2011.

[26] T. Schinko, H. Berger, W. Lee et al., "Transcriptome analysis of nitrate assimilation in Aspergillus nidulans reveals connections to nitric oxide metabolism," Molecular Microbiology, vol. 78, no. 3, pp. 720-738, 2010.

[27] S. Baidya, J. W. Cary, W. S. Grayburn, and A. M. Calvo, "Role of nitric oxide and flavohemoglobin homolog genes in Aspergillus nidulans sexual development and mycotoxin production," Applied and Environmental Microbiology, vol. 77, no. 15, pp. 5524-5528, 2011.

[28] S. Klaubauf, E. Inselsbacher, S. Zechmeister-Boltenstern et al., "Molecular diversity of fungal communities in agricultural soils from Lower Austria," Fungal Diversity, vol. 44, no. 1, pp. 65-75, 2010.

[29] G. F. Reed, F. Lynn, and B. D. Meade, "Use of coefficient of variation in assessing variability of quantitative assays," Clinical and Diagnostic Laboratory Immunology, vol. 9, no. 6, pp. 12351239, 2002.

[30] N. Lakhssassi, N. Elhajoui, J.-P. Lodter, J.-L. Pineill, and M. Sixou, "Antimicrobial susceptibility variation of 50 anaerobic periopathogens in aggressive periodontitis: an interindividual variability study," Oral Microbiology and Immunology, vol. 20, no. 4, pp. 244-252, 2005.

[31] M. R. Andersen, J. B. Nielsen, A. Klitgaard et al., "Accurate prediction of secondary metabolite gene clusters in filamentous fungi," Proceedings of the National Academy of Sciences of the United States of America, vol. 110, no. 1, pp. E99-E107, 2013.

[32] N. Khaldi, J. Collemare, M. Lebrun, and K. H. Wolfe, "Evidence for horizontal transfer of a secondary metabolite gene cluster between fungi," Genome Biology, vol. 9, no. 1, article R18, 2008.

[33] R. K. Pettit, "Small-molecule elicitation of microbial secondary metabolites," Microbial Biotechnology, vol. 4, no. 4, pp. 471-478, 2011.

[34] G. H. Talbot, "What is in the pipeline for gram-negative pathogens?" Expert Review of Anti-Infective Therapy, vol. 6, no. 1, pp. 39-49, 2008.

[35] J. Varga, M. Due, J. C. Frisvad, and R. A. Samson, “Taxonomic revision of Aspergillus section Clavati based on molecular, morphological and physiological data," Studies in Mycology, vol. 59, pp. 89-106, 2007.

[36] E. M. Boyd, "Patulin," Canadian Medical Association Journal, vol. 50, no. 2, p. 159, 1944.

[37] S. R. Parker, H. G. Cutler, and P. R. Schreiner, "Koninginin C: a biologically active natural product from Trichoderma koningii," Bioscience, Biotechnology, and Biochemistry, vol. 59, no. 6, pp. 1126-1127, 1995.

[38] R. W. Dunlop, A. Simon, K. Sivasithamparam, and E. L. Ghisalberti, "An antibiotic from Trichoderma koningii active 
against soilborne plant pathogens," Journal of Natural Products, vol. 52, no. 1, pp. 67-74, 1989.

[39] B. Zhang, L. P. Zheng, and J. W. Wang, "Nitric oxide elicitation for secondary metabolite production in cultured plant cells," Applied Microbiology and Biotechnology, vol. 93, no. 2, pp. 455466, 2012.

[40] J. H. Yu and N. Keller, "Regulation of secondary metabolism in filamentous fungi," Annual Review of Phytopathology, vol. 43, pp. 437-458, 2005.

[41] A. M. Calvo, R. A. Wilson, J. W. Bok, and N. P. Keller, "Relationship between secondary metabolism and fungal development," Microbiology and Molecular Biology Reviews, vol. 66, no. 3, pp. 447-459, 2002.

[42] J. H. Birkinshaw, A. Bracken, S. E. Michael, and H. Raistrick, "Studies in the biochemistry of micro-organisms; fuscin, a metabolic product of Oidiodendron fuscum Robak. Part 2. Derivatives and degradation products," The Biochemical Journal, vol. 48, no. 1, pp. 67-74, 1951.

[43] M. Jackson, J. P. Karwowski, P. E. Humphrey, W. L. Kohl, G. J. Barlow, and S. K. Tanaka, "Calbistrins, novel antifungal agents produced by Penicillium restrictum. I: production, taxonomy of the producing organism and biological activity," Journal of Antibiotics, vol. 46, no. 1, pp. 34-38, 1993.

[44] F. E. Koehn, "High impact technologies for natural products screening," Natural Compounds as Drugs Volume I, vol. 65, pp. 175-210, 2008. 

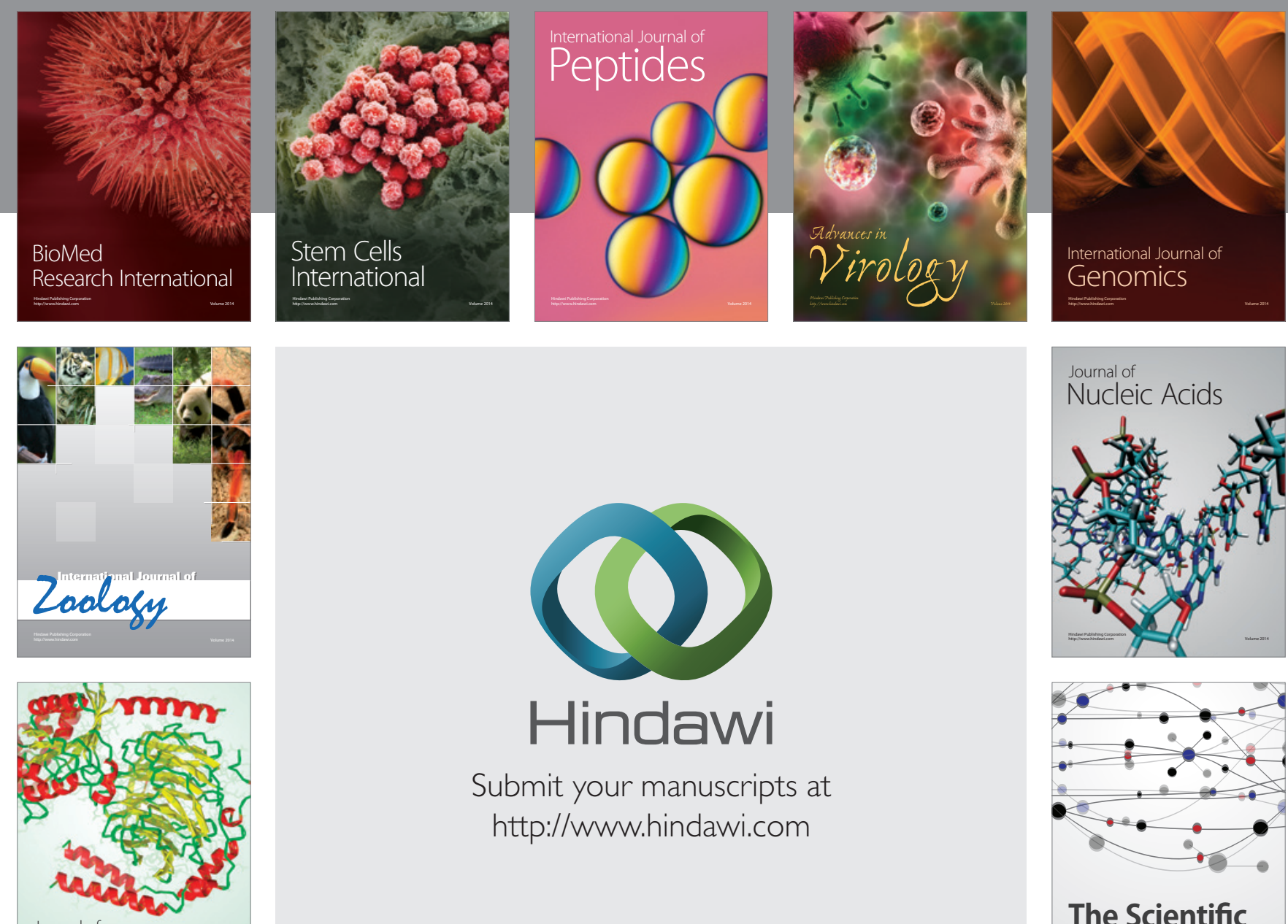

Submit your manuscripts at

http://www.hindawi.com

Journal of
Signal Transduction
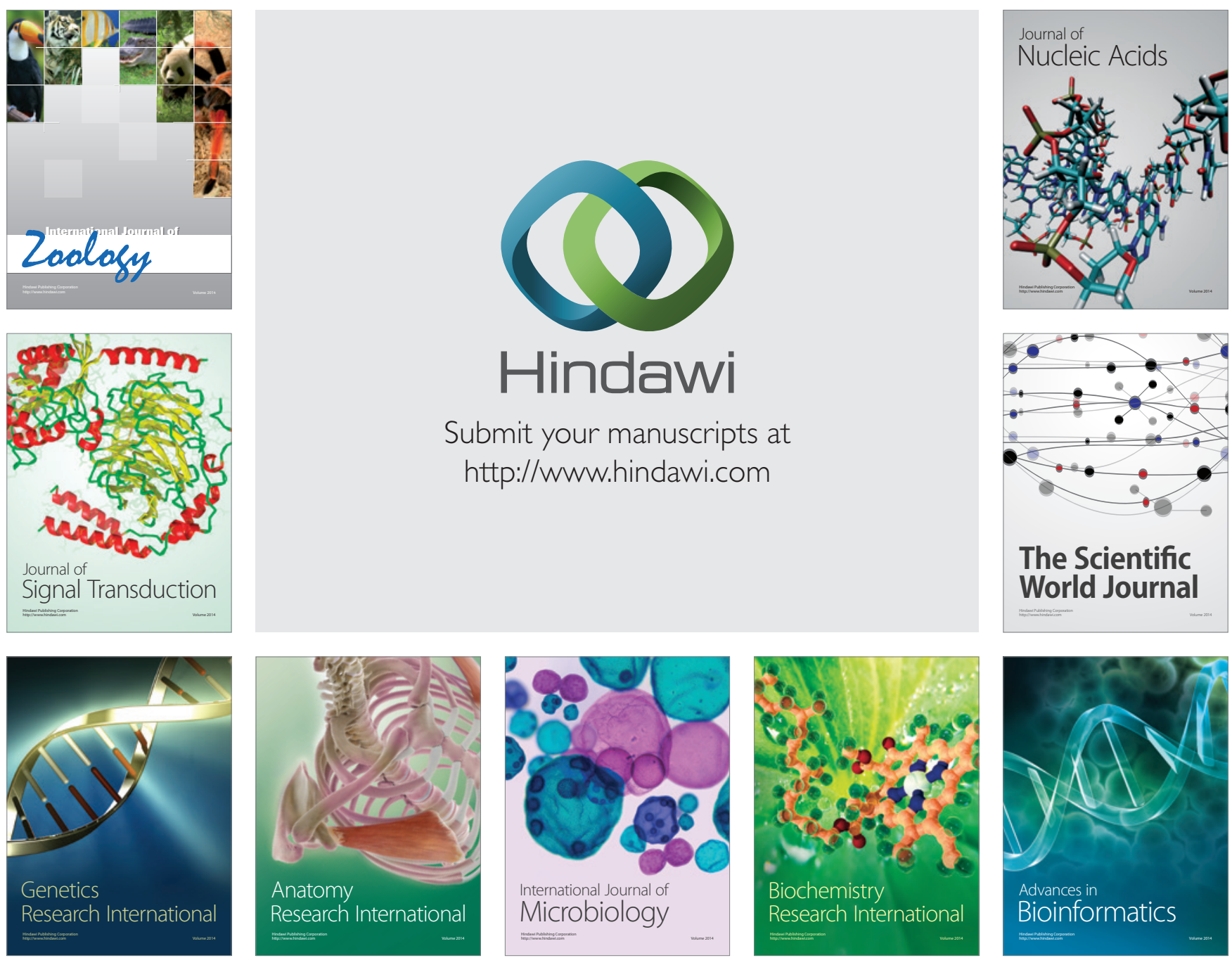

The Scientific World Journal
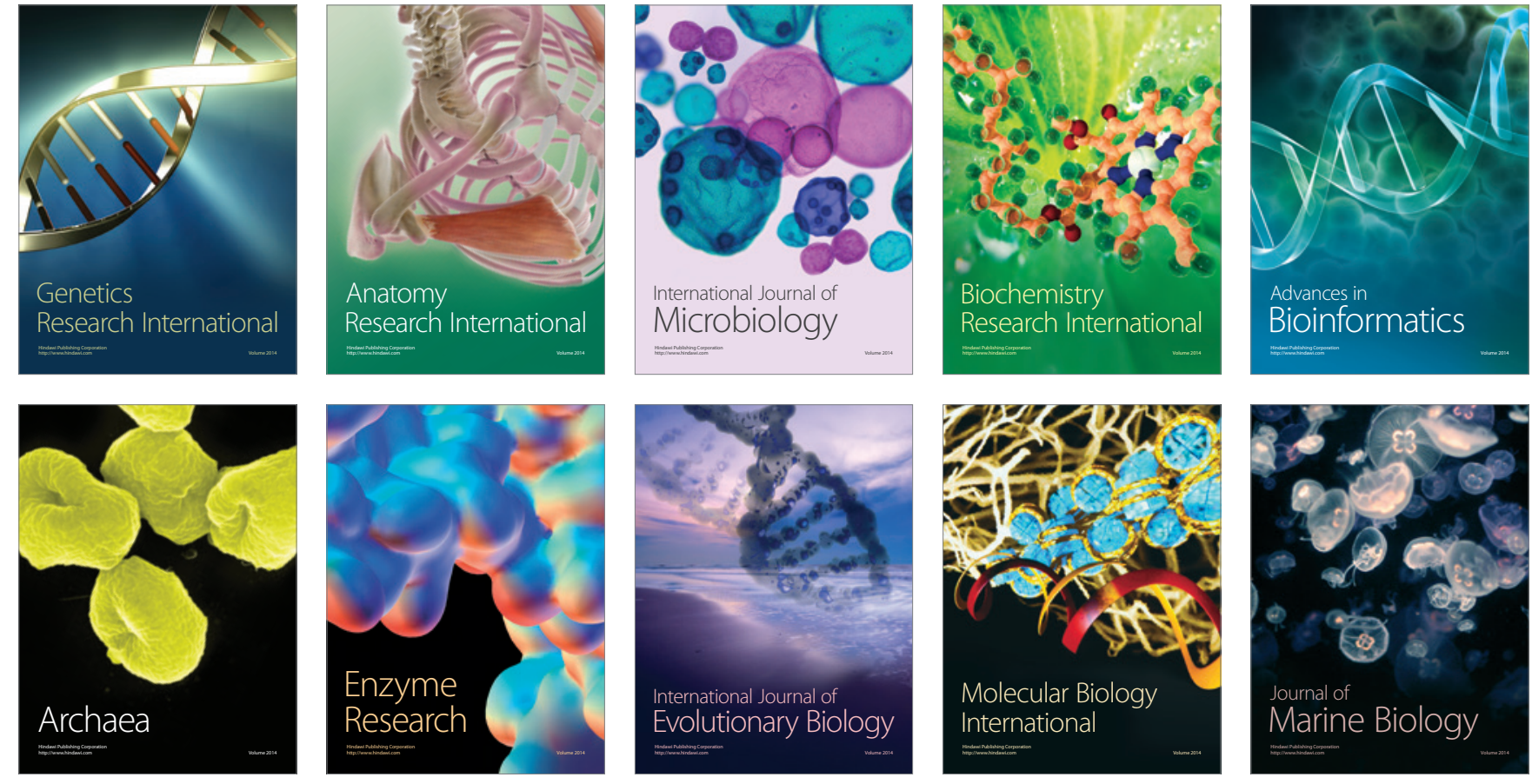